\title{
Communications
}

\section{Hanse-Preise 2022}

\section{Hanse-Preis für Intensivmedizin 2022}

gestiftet

von der Fresenius-Stiftung Bad Homburg

Dotation: EUR 5000

Der Gewinner/die Gewinnerin des HansePreises für Intensivmedizin wird in einem Vortragswettbewerb ermittelt. Berücksichtigt werden 2021 publizierte Originalarbeiten (peer reviewed, experimentell oder klinisch, deutsch oder englisch) aus den Bereichen der Intensiv- oder Notfallmedizin.

Hanse-Pflegepreis für die fachpraktische Arbeit 2022

gestiftet von der B. Braun Melsungen AG

Dotation: EUR 2000

\section{Hanse-Pflegepreis für die pflegewissen-} schaftliche Arbeit 2022

\section{gestiftet}

von der Vifor Pharma Deutschland GmbH

\section{Dotation: EUR 2000}

Die Gewinner der Hanse-Pflegepreise werden in einem Vortragswettbewerb ermittelt. Berücksichtigt werden veröffentlichte und unveröffentlichte Arbeiten aus den Bereichen der Intensiv- und Anästhesiepflege (auch Referate/Fach- und Jahresarbeiten im Rahmen der Fachweiterbildung).

\section{Hanse-Förderpreis für Konzepte zur Verbesserung der intensivmedizinischen Versorgungsqualität 2022}

gestiftet vom Wissenschaftlichen Verein zur Förderung der klinisch angewendeten Forschung in der Intensivmedizin e.V.

Dotation: EUR 3000

Der Förderpreis wird jährlich für innovative Projekte und Projektvorhaben vergeben, die auf eine nachhaltige Verbesserung der intensivmedizinischen und intensivpflegerischen Versorgungsqualität abzielen. Es werden vorzugsweise Projekte berücksichtigt, die anerkannte Instrumente des Qualitätsmanagements (PDCA-Zyklen) verwenden.

Hanse-Promotionspreis für klinische und experimentelle Forschung in der Intensivund Notfallmedizin 2022

gestiftet

von Achim Schulz-Lauterbach VMP GmbH

Dotation: je EUR 2000

Prämiert wird jeweils eine Promotionsarbeit aus den Bereichen Intensivmedizin oder
Notfallmedizin aus den Jahren 2020 und 2021 (Datum der Promotionsurkunde). Die Preisträger erhalten die Gelegenheit, ihre Arbeiten auf dem 32. Symposium 2022 vorzustellen.

\section{Hanse-Habilitationspreis 2022}

gestiftet

von der CytoSorbents Europe $\mathrm{GmbH}$

Dotation: EUR 10000

Ausgezeichnet werden bereits angenommene Habilitationsarbeiten aus den Jahren 2020 und 2021 (Datum der Habilitationsurkunde). Der Preisträger oder die Preisträgerin wird auf dem 32. Symposium 2022 vorgestellt und im Folgejahr als Referent oder Referentin zu einem Thema im Kontext der ausgezeichneten Habilitationsschrift eingeladen.

Einsendeschluss für alle Preise: 15. Dezember 2021 als PDF an: kontakt[at]wivim.org

Weitere Informationen finden Sie unter: wivim.org/preise

Auskünfte erteilt:

WIVIM e.V. Geschäftsstelle

Sandra Eylers

Sögestr. 48, DE-28195 Bremen

Tel.: +4930729820

E-Mail: Eylers.sandra[at]wivim.org

\section{Sujets d'actualité en ligne} www.bullmed.ch $\rightarrow$ Tour d'horizon

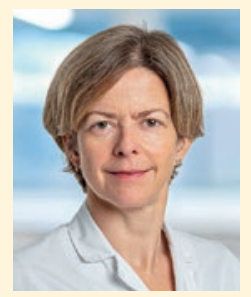

Entretien avec Prof. Dr méd. Maria Wertli, présidente Commission qualité de la Société Suisse de Médecine Interne Générale (SSMIG)

\section{Indicateurs de qualité - Avantages et limites}

La Commission qualité de la SSMIG présente pour la première fois les nouveaux indicateurs de qualité pour le traitement stationnaire.

Editorial de Sandra Ziegler, directrice des EMH Editions médicales suisses

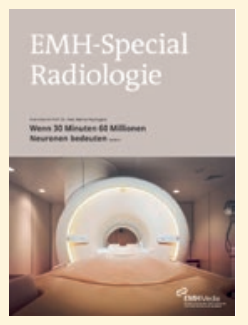

\section{EMH-Special: une nouvelle série de publications}

Des articles sur des sujets captivants, de précieuses informations pour la pratique médicale - voici ce qui caractérise la nouvelle série de publications «EMH-Special».

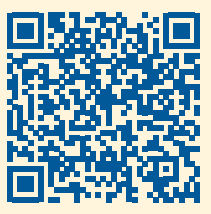

